\section{Trends in Linguistics}

Studies and Monographs 244.1

\section{Editor}

Volker Gast

\section{Founding Editor}

Werner Winter

Editorial Board

Walter Bisang

Hans Henrich Hock

Heiko Narrog

Matthias Schlesewsky

Niina Ning Zhang

Editor responsible for this volume

Matthias Schlesewsky

De Gruyter Mouton

\section{Frequency Effects in Language Learning and Processing}

edited by

Stefan Th. Gries

Dagmar Divjak

De Gruyter Mouton 


\title{
Are effects of word frequency effects of context of use? An analysis of initial fricative reduction in Spanish*
}

\author{
William D. Raymond and Esther L. Brown
}

The connection between frequency of form use and form reduction in language has been widely studied. After controlling for multiple contextual factors associated with reduction, word frequency, which reflects a speaker's cumulative experience with a word, has been reported to predict several types of pronunciation reduction. However, word frequency effects are not found consistently. Some studies have alternatively reported effects on reduction of the cumulative exposure of words to specific reducing environments or measures of contextual predictability. The current study examines cumulative and contextual effects of reducing environments, as well as non-contextual frequency measures, on the reduction of wordinitial /s/ in a corpus of spoken New Mexican Spanish. The results show effects of non-cumulative factors on reduction, argued to occur on-line during articulation. There are also effects of the cumulative exposure of words to specific reducing environments and of contextual predictability, but not of the cumulative experience with a word overall (word frequency). The results suggest representational change in the lexicon through repeated exposure of words to reducing environments and call into question proposals that frequency of use per se causes reduction.

\section{Introduction}

Word frèquency can be considered to reflect the relative cumulative experience that speakers have with words. The connection between word fre-

* We would like to thank Stefan Th. Gries for his help with the analysis of our data using $\mathrm{R}$ and acknowledge the constructive comments of Dr. Gries and three anonymous reviewers of this paper. We would also like to thank Lise Menn for useful comments on an earlier draft of the paper. This work was supported in part by Army Research Office Grant W911NF-05-1-0153 and Army Research Institute Contract DASW01-03-K-0002 to the University of Colorado. 
quency and reduction in language has been widely studied, with the result quency and reduction in language has beenronic change and that word frequency has been implicated in both diachronic change and synchronic production variation. Investigations of the processes of sound change in language going back over a century have noted that more frequent words are shorter and change more quickly than less frequent words (Schuchart 1885; Zipf 1929). In studies of synchronic pronunciation variation, evidence has been offered that higher word frequency is associated with more word reduction in speech production, as measured by both categorical measures of segment reduction or deletion (Bybee 2001, 2002; Krug 1998; Jurafsky et al. 2001; Raymond, Dautricourt, and Hume 2006) and also continuous durational shortening (Gahl 2008; Jurafsky et al. 2001; Pluymaekers, Ernestus, and Baayen 2005) and some acoustic parameters (Ernestus et al. 2006; Myers and Li 2007). Given prior results, it is widely assumed that frequency of word use contributes to reductive processes, although the mechanism by which it does so is unclear.

Word frequency is, of course, not the only correlate of reduction. Studies of word frequency effects on pronunciation variation have commonly controlled many factors that contribute to reductive phenomena, including trolled many factors that contribute to reductive phenol context, prosodic environment, speech rate, sociolinguistic factors, and even probabilistic variables other than word frequency. Even after controlling for multiple factors contributing to reduction, word frequency has usually been reported to predict pronunciation reduction by at least some measures; however, frequency effects are not ubiquitous. For example, Pluymaekers et al (2005) found that word frequency affected reduction of affix form and duration for most but not all of the morphologically complex Dutch words they studied. Similarly, some of the high-frequency function words examined by Jurafsky et al. (2001) had low rates of reduction, despite their high frequency and a control for phonological context. Finally, Cohn et al. (2005) found no effect of word frequency on durational shortening of homophones, although Gahl (2008) did.

Failure to find effects consistently of word frequency on production variation, within studies or between comparable studies, has been attributed to methodological differences, such as sample size (see Gahl 2008) or to the set of factors considered in the study. Indeed, one class of factors that has not commonly been included in reduction studies in conjunction with word frequency is the likelihood that a word occurs in discourse contexts in a phonological environment that promotes reduction. The importance of this type of cumulative contextual measure has been noted (Bybee 2001, 2002; Timberlake 1978). For example, the rate of word-final t/d deletion in English is lower for words that are more likely to occur in the context of a following vowel in speech (Guy 1991; Bybee 2002). Similarly, reduction rates of word-initial [s] in Spanish are higher for words that are more likely to occur in the context of a preceding non-high vowel in speech (Brown 2004, 2006). However, other probabilistic measures, such as word and phone frequencies and predictabilities, were not always controlled in these studies along with the cumulative reducing context variables. Conversely, studies finding frequency effects have not controlled the likelihood of a word occurring in a reducing environment. For example, Jurafsky et al. (2001) found effects of frequency on segment deletion and durational shortening of final $\mathrm{t} / \mathrm{d}$ in content words, but their study did not control the likelihood of words occurring before following consonants, an extralexical environment promoting deletion. By comparison, in a study of word-internal $\mathrm{t} / \mathrm{d}$ deletion, thus testing words for which phonological context of $\mathrm{t} / \mathrm{d}$ is constant, Raymond et al. (2006) found no effect of frequency on deletion after controlling for predictability of the $t / d$ word from the preceding and following words.

If word frequency plays a causal role in reductive processes, how might it affect reduction? In some usage-based theories of language (Bybee 2001, 2002) the effect of word frequency on sound change is explained as the result of automation of production processes. Production automation is claimed to result in more casual, more reduced forms, which will ultimately be registered as change in lexical representation. Automation is signaled by production speed, and frequent words can certainly be accessed more quickly than infrequent words (Balota et al. 2004; Forster and Chambers 1973). It could be that access speed has a direct effect on the articulation of words; however, an explanation based simply on how often a word is used would seem to entail that reductive change should occur uniformly across the word and not merely on certain segments or syllables, contrary to observations of lexical change (see Pluymaekers et al. 2005). The fact that reduction in frequent words is not uniform suggests there is an influence of lexical structure and discourse environments on reductive processes, leading to differential articulatory effects, automation processes, and, ultimately, reduction. Identifying any effect of word frequency on reduced pronunciation at articulation independent of reducing environments thus depends on controlling the factors leading to on-line articulatory reduc- 
tion, cumulative measures of exposure to reductive environments, and measures of contextual predictability.

The current study examines the role of word frequency in reduction by examining a specific reduction phenomenon, word-initial [s] (s-) lenition, in the spoken Spanish of New Mexico. Many modern dialects of Spanish exhibit synchronic variation in production of [s] from full $/ \mathrm{s} /$ to $[\mathrm{h}]$ or even deletion (ø), either syllable finally (Terrell 1979; Lipski 1984; Brown, E. K. 2008; File-Muriel 2009) or syllable initially (Brown and Torres Cacoullos 2002). For syllable initial reduction the segmental context favoring reduction is a neighboring non-high vowel (/a, e, o/). Non-high vowels both preceding and following [s] have been found to increase the likelihood of reduction (Brown 2004, 2006), presumably because the non-high vowels' lower tongue height increases the likelihood that the alveolar target of [s] will be undershot. New Mexican Spanish is one dialect in which syllable-initial [s], including word-initial [s], may undergo lenition to [h] or even be deleted (e.g., tuve que [h]alir, for tuve que salir, "I had to leave"). In a study of s- reduction in this dialect, Brown (2006) found that the likelihood with which a word occurs in a non-high vowel environment predicts s- reduction. Interestingly, in her study the rate of reduction was also higher in words with high frequency than in words with low frequency, although other probabilistic measure were not controlled.

In the current study whether word frequency plays an independent role in on-line s- reduction is addressed by controlling both word frequency and frequency of occurrence of a word in phonological environments known to promote articulatory reduction of [s-]. The effects of other probabilistic measures are also assessed, to determine whether they contribute to s- reduction. Both intra- and extra-lexical phonological contexts are controlled, and comparison of their effects is used to determine to what extent reduction can be attributed to lexical representations or on-line articulatory processes.

\section{Data and Methods}

The data used in this study largely come from the materials of The New Mexico-Colorado Spanish Survey (NMCOSS) (Bills and Vigil 2008). The NMCOSS project, initiated in 1991, documents, via interviews with 350 native speakers, the Spanish language spoken throughout the state of New Mexico and sixteen counties of southern Colorado (Bills and Vigil 1999). The NMCOSS interview corpus was collected by trained field workers who tape-recorded interviews involving both controlled elicitation and guided conversation (Vigil 1989). Each NMCOSS interview averaged three and a half hours in length, beginning with compilation of personal information regarding the consultant and followed by specific linguistic elicitation and free conversation. The interviews were subsequently orthographically transcribed.

The dataset for this study was created from the free conversation portions of the interviews of a subset of 16 men and 6 women selected at random from the NMCOSS study corpus, as well as two additional interviews with male native speakers of the same New Mexican dialect, for a total of 24 consultants. The data from one of the additional consultants was taken from an unplanned, self-recorded conversation. The data of the other additional consultant was extracted from a recorded conversation making up part of the Barelas study (conversational data of the Spanish spoken in the Barelas neighborhood of Albuquerque, NM), collected in recorded sociolinguistic interviews by students of a Spanish graduate course at the University of New Mexico in 2001. Although all consultants were native speakers of Spanish, most also had English proficiency, and there is a substantial amount of code switching and borrowing in the interviews. About $4 \%$ of the interview words were English words. The token dataset analyzed consists of all [s]-initial Spanish words (s- words) extracted from the set of words spoken by consultants in the conversations with the 24 consultants. The final dataset contained 2423 tokens (from 209 types) of [s]-initial Spanish word tokens. The phonetic realization of all $/ \mathrm{s} /$ phones in each token of these words was transcribed as perceptually reduced ([h] or ø) or unreduced ([s]) by one of the authors (EB), with reliability checks from native speakers.

The transcribed interview speech of the 22 NMCOSS consultants was used for frequency counts of phone and word units and bigrams. Both interviewer and consultant Spanish utterances in the recorded, transcribed conversations with these consultants were used to calculate unit frequencies. Word unit counts were compiled in five categories of units in the corpus subset: (1) whole Spanish word productions (word) (2) phrase boundaries (based on punctuation) and utterance (speaker) boundaries (pause); (3) partial word productions (cutoff); (4) hesitations and fillers (e.g., "uh"; filler); and (5) English words (english). All backchannel utterances (e.g., "oh" and "uh-huh") and sequences that could not be clearly understood during transcription (and were marked in the transcription as unclear) were excluded from the word unit counts. Word bigram counts were 
made for all pairs of word units, producing statistics for each word that reflect how often it occurred after each other Spanish word, after a cutoff, after a pause, after a filler, and after an English word. Phone unit counts were calculated from word unit and word bigram counts for all phones in Spanish words. The phone unit counts were tallied separately for phones at word boundaries, phones at utterance boundaries, and all other phones within words. Phone bigram counts were tallied separately for phone pairs across word boundaries, phones adjacent to pauses and phone pairs within words. Phones adjacent to cutoffs, fillers, and English words were excluded from the phone and phone bigram counts. After exclusions, counts of word units and phones in the speech of the NMCOSS subset of speakers resulted in frequencies for about 75,000 words units and 280,000 phones of speech.

The word and phone counts from the speech of the corpus subset were used to create a database of word and phone statistics that includes the measures in Table 1.

Table 1. Measures included in the database of word and phone statistics

1. Word unit frequency per million of each word unit in the corpus subset (word frequency);

2. Word bigram frequency per million of each word unit and the word unit preceding it (word bigram frequency);

3. Predictability of each word unit from the word unit preceding it, calculated as the bigram frequency of the s- word divided by the frequency of the preceding word unit. (preceding word predictability, $\mathrm{P}\left(w_{i} \mid w_{i-1}\right)$ );

4. Predictability of each word unit from the word unit following it (following word predictability, $\left.\mathrm{P}\left(w_{i} \mid w_{i+1}\right)\right)$;

5. Frequencies of all phone units in words (phone frequency);

6. Frequencies of all phone bigrams consisting of a word phone and the phone unit preceding it (phone bigram frequency);

7. Predictability of all phone units in words from the phone unit preceding it ( preceding phone predictability, $\mathrm{P}\left(\varphi_{i} \mid \varphi_{i-1}\right)$ );

8. Predictability of a phone unit from the phone unit following it (following phone predictability, $\left.\mathrm{P}\left(\varphi_{i} \mid \varphi_{i+1}\right)\right)$.

Using the interview transcriptions (with phonetic annotation of the realization of initial /s/ phones) and the word and phone statistics described in Table 1, the s- word tokens from the 24 consultants used for the study were coded for the ten variables in Table 2
Table 2. Variables coded for each token in the s- word dataset

1. Realization of initial /s/ in the consultant's speech ([s] = unreduced; $[\mathrm{h}]$, $\varnothing=$ reduced);

2. Favorability of preceding phone context for s- reduction (yes for preceding non-high vowels, no for all other preceding phone units);

3. Frequency with which the phone preceding s- occurs before s- in the corpus, that is, the phone bigram frequency of s- (based on the word orthography, with phrase- and utterance-initial words coded as preceded by a pause);

4. Proportion of times in the corpus that the s- word has a preceding context favorable for fricative reduction, which is the proportion of tokens for an s- word type that are preceded by a non-high vowel (Frequency in a Favorable Context, or FFC);

5. Favorability of the phone following s- for s- reduction (yes for following non-high vowels, no for all other following phones);

6. Log of s- word frequency per million;

7. Identity of tokens of the very frequent clitic se used as a 3 rd person singular reflexive pronoun, a $3 \mathrm{rd}$ person singular indirect object, and in impersonal constructions ( $y e s$ for se tokens, no for all other tokens);

8. Hapax words in the corpus (yes for words with only a single token in the dataset, no for all words with more than one token);

9. Stress on s- syllable (stressed if lexical stress on primary syllable, unstressed if lexical stress on non-initial syllable or if the word is a clitic or function word);

10. Predictability of the s- word from the preceding word unit, $\mathrm{P}\left(w_{s-\mid} \mid w_{s-1}\right)$.

As an illustration of the measures in Table 2, consider the excerpt from the corpus transcription in (1). The s- word sobrino in the token in (1) occurs 11 times in the NMCOSS corpus, giving it a frequency per million of 146 and a $\log$ frequency of 2.17 . The preceding word bigram in this token is mi sobrino, which has a frequency in the corpus statistics of 6 , and the frequency of the word preceding the s- word, $m i$, is 485 , so that the predictability of sobrino from $m i$ is $6 / 485=.0124$. The s- of sobrino in this token is followed (word-internally) by the non-high vowel /o/, which is a context hypothesized to favor s- reduction. However, the vowel preceding s- is the high vowel /i/ in $m i$, which is hypothesized not to favor reduction. Overall in the corpus the word sobrino occurs after a non-high vowel $(/ \mathrm{o} / \mathrm{a} / \mathrm{a} /$, or $/ \mathrm{e} /)$ only once, giving sobrino a FFC of $1 / 11=.091$. The frequency with which /i/ precedes /s/ at a word boundary in the corpus is 407, and the $\log$ of this frequency per million phones is 2.61 . 
(1) ... a mi sobrino, porque yo... ... to my nephew, because I...

The variables $2-10$ in Table 2 were used in regression analyses as predictors of the outcome variable, the binary variable 1 (phonological realization of s-), coding s- reduction. The predictor variables provide control over many of the factors that have been shown generally to influence reduction in speech. Specifically, reduction is higher in unstressed syllables than in stressed syllables (de Jong 1995). Reduction is also influenced by intra- and extra-lexical phonological contexts, which vary according to the variable under investigation (Brown 2006; Raymond, Dautricourt, and Hume 2006, Rhodes, R. A. 1992). In this study we consider only the phone context preceding s- words and the phone following s-. Non-high preceding vowels in both of these contexts have been shown to encourage s- reduction (Brown 2005). Effects of phone frequencies on s- reduction have not previously been investigated. More likely word and phone combinations are often associated with higher reduction (Jurafsky et al. 2001; Krug 1998). Note that frequency variables are skewed, with a few very high frequency tokens and many low frequency tokens. By taking the log of the frequency, this disparity is lessened (Gries 2009). The word se was chosen for identity coding because it is highly frequent (comprising $12.9 \%$ of the tokens), unstressed, and has a high reduction rate $(.228)$. It is the only clitic form in the dataset, and clitics are known to behave differently from other words (Gerlach and Grijzenhout 2000). Hapax forms were coded specially because single occurrences of words in the limited speech sample of this corpus may not provide a reliable estimate of the cumulative experience of speakers with factors approximated using the probabilistic variables in Table 2, especially those variables that are calculated across extra-lexical phonological contexts.

The predictor variables for the study thus include four probabilistic measures: (1) s- word frequency; (2) the log frequency of the phone preceding the s-; (3) the predictability of the s- word from the word unit preceding it in context; and (4) the proportion of times that the s- word is preceded in production contexts by a non-high vowel (FFC). The first two measures are simple frequencies, whereas the last two are predictability measures. In addition, FFC and the frequency of the preceding phone consider the preceding phone as the context, whereas $\mathrm{P}\left(w_{s-} \mid w_{s--1}\right)$ uses as context the preceding word. These variables thus allow us to look separately at the effects of the type of probabilistic measure (frequency or predictability)
Table 3. Independent variables categorized by type of probabilistic measure and context unit size

\begin{tabular}{lll}
\hline & \multicolumn{2}{l}{ Type of probabilistic measure } \\
\cline { 2 - 3 } Context unit size & Frequency & Predictability \\
\hline Word & Word frequency & $\mathrm{P}\left(w_{s-} \mid w_{s-1-1}\right)$ \\
Phone & Frequency of preceding phone & FFC \\
\hline
\end{tabular}

and context unit size (word or phone), as summarized in Table 3. Effects of word and phone unit probabilities on reductive processes have been found in previous studies, and FFC, which is a specific measure of exposure of words to a reductive environment, has also been implicated in s- reduction. However, these three measures have not previously been considered together in a study of reduction.

In addition to assessing the effects of frequencies and predictabilities, a second goal of the current study is to examine the contributions to sreduction of factors that reflect cumulative experience with a word (including word frequency) and those that do not, as well as the effects of variables that refer to the extra-lexical (preceding) production context and those that are purely word internal, and thus are not context dependent. The independent variables chosen can be categorized in terms of these two dimensions, as shown in Table 4. Effects of the variables reflecting cumulative experience are taken to indicate a lexical source of reduction; effects of variables that are context dependent largely reflect an influence of articulation on reduction, either during production or as registered in lexical representation.

Note that other factors that have been shown to influence reduction in speech are not examined in this study, in particular speech rate (FosslerLussier and Moran 1999; Jurafsky et al. 2001), syntactic probabilities (Gahl and Garnsey 2004), and predictability from semantic or broader discourse context (Bard et al. 2000; Fowler and Housum 1987). Although these factors may also predict s- reduction, they seem unlikely exclusively to explain any effects of the articulatory and probabilistic variables with which we are concerned in our analyses. Moreover, the focus of the current study is to examine the scope and nature of variables implicated specifically in s- reduction, which can be assessed with the variables chosen for analysis. 
Table 4. Independent variables categorized by cumulative experience and context dependence

\begin{tabular}{lll}
\hline \multirow{2}{*}{ Context dependence } & Cumulative experience \\
\cline { 2 - 3 } Yes & FFC, $\mathrm{P}\left(w_{s-1} \mid w_{s-1}\right)$, & No \\
\hline \multirow{2}{*}{ No } & Frequency of preceding phone & $\begin{array}{l}\text { Preceding favorable } \\
\text { context }\end{array}$ \\
& Word frequency & $\begin{array}{l}\text { Stress, Following } \\
\text { favorable context }\end{array}$ \\
\hline
\end{tabular}

\section{Results}

The data were analyzed with the $\mathrm{R}$ statistical package using logistic regression, with realization of s- as reduced or unreduced as the dependent variable. Analyses were performed on all s- data, as well as on some subsets of s- data, in order to examine the effects of one variable on a particular subcategory of s- words in one case, as described later in this section.

The model likelihood ratio for the model identified in the analysis was 341.64 (d.f. $=17 ; \mathrm{p}<.0001$ ). Using the mean of the predicted probabilities as the cutoff, classification accuracy for the model was $83.6 \%$. However, the overall correlation for the model was not high (Nagelkerke $r^{2}=.223$ ). The proportion of reduced tokens is low (.164), so that the null model that assumes no reduction would have comparable accuracy, although little explanatory power for the phenomenon. Additional accuracy would perhaps be achieved with the inclusion of other factors associated with reduction that were not included in the current analysis, especially speech rate.

The results of the analysis of the complete dataset are shown in Table 5, along with the odds ratios for the significant predictors. All measures 2-10 of Table 2 were used in this analysis except the log of the preceding phone frequency, because only three phones can precede s- in tokens preceded by a favorable reducing environment (i.e., the non-high vowels /e, a, o/, all of which have high frequencies), making the continuity of the variable in this environment questionable.

As shown in the table, there was a main effect of both the preceding and following phonological contexts of s-, with non-high vowels predicting higher reduction rates in both environments. When an s-word is preceded by a non-high vowel, it is 2.41 times more likely to be reduced
Table 5. Results of analysis of the complete dataset $(\mathrm{N}=2423)$.

\begin{tabular}{lll}
\hline & $p$ & $\begin{array}{l}\text { Odds Ratio } \\
\text { Effect }\end{array}$ \\
\hline Preceding favorable context $=$ yes & 0.0302 & 2.41 \\
Following favorable context $=$ yes & $<.0001$ & 3.03 \\
Syllable stress $=$ yes & 0.0235 & 1.59 \\
se $=$ yes & 0.0019 & 4.55 \\
$\mathrm{P}\left(w_{s-} \mid w_{s-}-1\right)$ & 0.0183 & 1.01 \\
FFC & 0.0457 & 2.33 \\
Preceding favorable context X se & 0.0310 & N.A. \\
FFC X Log word frequency & 0.0036 & N.A. \\
\hline
\end{tabular}

than when it is preceded by a high vowel, a consonant, or a pause. Similarly, when an s- is followed in a word by a non-high vowel, it is 3.03 times more likely to be reduced than when it is followed by a high vowel or a glide $(/ \mathrm{i}, \mathrm{u}, \mathrm{j}, \mathrm{w} /)$. There was also a main effect of the stress variable on reduction in the complete dataset, with no lexical stress on the initial syllable of an s- word making it 1.59 times more likely to be reduced than if the initial syllable has stress. After controlling for phonological context, there was a main effect of the cumulative contextual variable FFC, reflecting the fact that words in the highest quartile of FFC were 2.33 times more likely to be reduced than words in the lowest quartile. There was also an effect of the cumulative variable word predictability on reduction. Although significant, the effect of s- word predictability from the preceding word was very small, with words in the highest quartile of predictability only $1 \%$ more likely to be reduced than words in the lowest quartile of predictability. In addition there was a main effect of se word identity, with se 4.55 times more likely to be reduced than other words, even after controlling factors of se that contribute to its reduction. There was no main effect in the dataset on reduction of the non-contextual cumulative variable word frequency or of hapax words.

There were also two significant interactions in the analysis. The first interaction involved preceding favorable context and se word identity. As shown in Figure 1, the phonological context preceding an s- word has a greater effect on reduction for se than for other s- words. The interaction suggests a strong influence of articulatory environment on reduction of this clitic. 


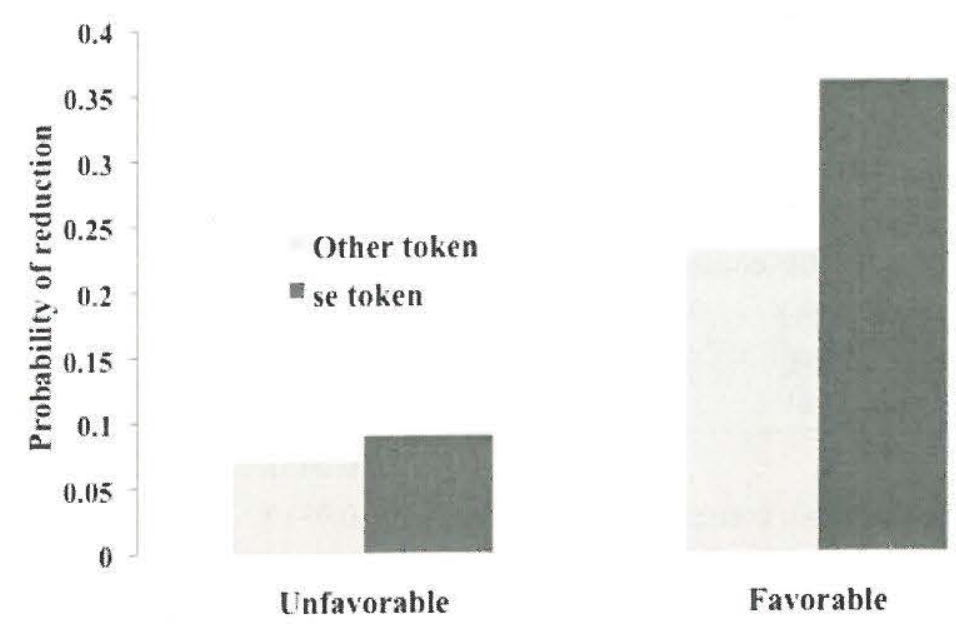

Preceding phonological environment

Figure 1. Interaction of preceding favorable context and se word identity on reduction of s-

The second interaction involved word frequency and FFC. Note that FFC and word frequency are related measures, because FFC is defined as the number of tokens of a word occurring in reducing environments out of the total number of tokens of the word in the dataset, or its frequency of occurrence. An interaction of FFC and word frequency is suggested by the fact that there is a correlation between FFC and reduction rate for high frequency words $(\mathrm{r}=.36)$, but not low frequency words $(\mathrm{r}=.12)$, as shown in Figure 2.

To test the effect of preceding phone frequency on reduction of s-, an analysis was done of a subset of the data that included only tokens that were not preceded by a non-high vowel, that is, the set of tokens not in a discourse environment favorable for reduction. Tokens in an environment favorable to reduction were not included in the analysis because there are only three non-high vowels that comprise a preceding favorable environment. The number of tokens in the reduced dataset was 1177 , of which only $7.2 \%$ were reduced.

In a logistic regression on this subset of the data including all measures $2-10$ of Table $292.9 \%$ of the tokens were correctly categorized by the model. The analysis showed an effect of a following favorable environment $(p=.0054$, odds ratio effect $=2.46)$ and a small effect of stress $(p=.0272$,

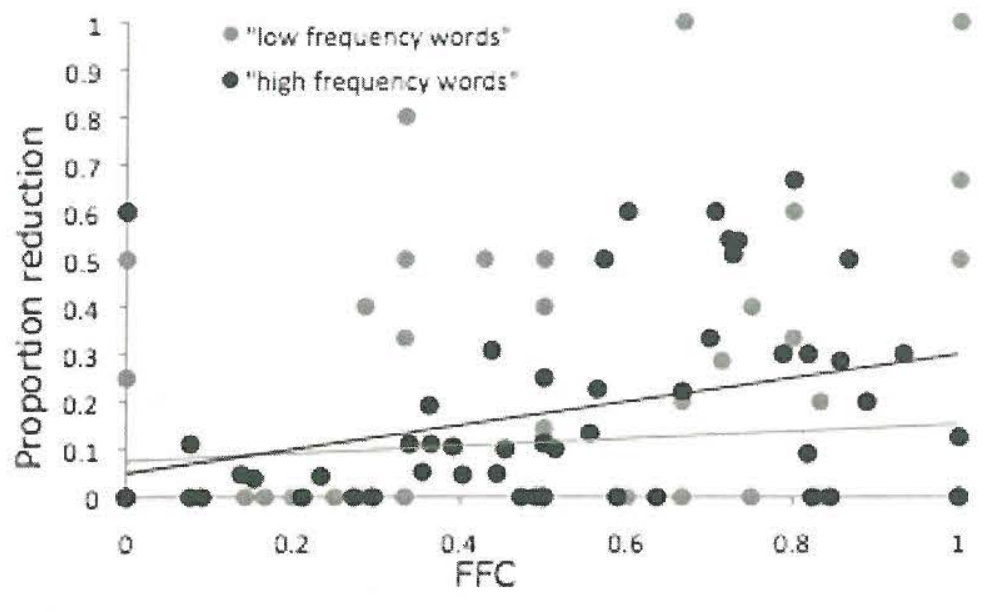

Figure 2. Interaction of FFC and reduction for high and low frequency s- words

odds ratio effect $=1.05$ ). There was also a significant interaction of frequency of preceding phone and predictability of the s- word from the preceding word; however, the interaction was complex and will not be interpreted here. There was no significant effect of preceding phone frequency on reduction.

\section{Discussion}

The results for the complete dataset show effects on reduction of both extra- and intra-lexical factors, as well as variables that reflect speakers' cumulative experience with words and those that are a function of the context in which words are produced. However, not all variables in the analyses significantly predicted s- reduction, and there were interactions among some variables. The pattern of results allows us to draw conclusions about the sources of reductive influences, and, specifically, the role of word frequency in s- reduction.

There were strong effects on s- reduction of the non-cumulative intraand extra-lexical variables involving phonological contexts. The tendency for non-high vowels that precede s- in the discourse context or that follow $s$ - in a word's lexical form to encourage reduction suggests an on-line articulatory effect of phonological context. Tongue lowering during the articulation of $/ \mathrm{s} /$ before and/or after articulation of a non-high vowel is 
a likely explanation for why the /s/ target was sometimes not achieved in these contexts. In the context of high vowels, on the other hand, tongue height in the vowel would facilitate $/ \mathrm{s} /$ articulation. Articulations of $/ \mathrm{s} /$ were also more likely to be reduced in unstressed syllables (de Jong 1995), which can account for the greater reduction rate for /s/ in lexically unstressed syllables over lexically stressed syllables.

There were also effects of cumulative context variables. The effect of the predictability of the s- word from the preceding word was small. However, the effect of FFC was robust and confirms earlier findings that FFC encourages reduction in studies that did not control other probabilistic factors (Brown 2004, 2006). The effect of FFC indicates that the cumulative experience of words in reducing phonological contexts of non-high preceding vowels results in a greater likelihood of reduction than context of use alone can explain. The effect suggests that reduction of s- reflects changes in the lexical representations of words through cumulative experience with these words in reductive production contexts. Because speakers have more limited experience with low frequency words than with high frequency words, the low frequency words show less of an effect of cumulative experience on their representations than do high frequency words.

The influence of factors defined by cumulative phonological context on $\mathrm{s}$ - reduction is compatible with an incipient process of lexicalized reduction of s- to [h] or ø. Lexical changes have not resulted in deterministic allophonic alternation in New Mexican Spanish: Most s- words continue to exhibit production variation in the data examined. The continued cumulative effects of discourse context and lexical structure on individual words may eventually result in a lexical distribution of phone variants, as happened, starting in Medieval Spanish, with words derived from Latin words beginning with /fV/ (FV- words). In Modern Spanish some FVwords begin with [f] (e.g., fe "faith" from L. FIDES and fácil "easy" from L. FACILIS) and some have an empty onset ([ø], spelled $h$ ) (e.g., hablar "to talk, to speak" from L. FABULARE and hijo "son" from L. FILIUS). Brown and Raymond (2010) have shown that the distribution of $/ f \mid \sim[\varnothing]$ in FV- words in Spanish is predicted by, among other variables, the likelihood that the words occur after a non-high vowel, the FFC variable also shown to predict the reduction of $/ \mathrm{s} /$ in $\mathrm{s}-$ words in the current synchronic study.

After taking into account the effects on s- reduction of non-cumulative phonological variables and the predictability variables involving word and phone context, there was no influence on reduction in the complete dataset or the subsets tested of preceding phone frequency or s- word frequency.
The failure to find any robust effects of the non-contextual word and phone unit probabilities after controlling the contextual variables suggests that speakers are sensitive to how often a word occurs in environments that encourage reduction, but not measurably to non-contextual probabilistic measures of use. Consequently, an s- word's frequency did not predict /s-/ reduction.

How can the failure to find a significant effect of word frequency on sreduction in datasets analyzed be reconciled with other studies, in which word frequency effects on a range of reductive processes have been reported? As noted, in most of these studies the likelihood of a word occurring in a reducing environment was not controlled. With respect to phonological context, the environments promoting reduction are generally identifiable, and tests of their importance could be readily made. However, other variables not examined in this study may also promote reduction differentially across the word frequency range. For example, higher rates of speech are associated with reduction, and words may differ in their likelihood of being produced at high speech rates. In addition to a direct effect of speech rate on reduction, higher frequency words may, in particular, be more likely to be produced in contexts with higher speech rates than lower frequency words. Because faster speech rates may encourage reduction, high frequency words would thus have a higher probability of occurring in this reducing environment. In support of this possibility, Gahl (2008) found that, after controlling other factors influencing word duration, a higher speaking rate in the region following target words that are members of homophone pairs predicted shorter durations of the higher frequency member of the pair. Word frequency remained a significant predictor in the Gahl model; however, note that the likelihood of a word occurring in a high speech rate region, a context promoting reduction, was not controlled. It remains to be tested whether variability in the likelihood that words occur in reducing environments defined by non-phonological variables such as speech rate can also predict reduction and eliminate word frequency effects.

\section{References}

Balota, David. A., Cortese, Michael J., Sergent-Marshall, Susan D., Spieler, Daniel 2004 H. and Yap, Melvin J.

2004 Visual word recognition of single-syllable words. Journal of Experimental Psychology: General, 133, 382-316. 
Bard, Ellen G., Anderson, Anne H., Sotillo, Catherine, Aylett, Matthew, DohertySneddon, Gwyneth and Newlands, Alison

2000 Controlling the intelligibility of referring expressions in dialogue. Journal of Memory and Language, 42, 1-22.

Bills, Garland and Vigil, Neddy

1999 Ashes to ashes: The historical basis for dialect variation in New Mexican Spanish. Romance Philology, 53, 43-67.

Bills, Garland and Vigil, Neddy

2008 The Spanish Language of New Mexico and Southern Colorado: A Linguistic Atlas. Albuquerque: University of New Mexico.

Brown, Earl K.

2008 A Usage-based Account of Syllable- and Word-final /s/ Reduction in Four Dialects of Spanish. Doctoral Dissertation, University of New Mexico.

Brown, Esther L.

2004 Reduction of syllable-initial /s/ in the Spanish of New Mexico and Southern Colorado: A usage-based approach. Doctoral Dissertation, University of New Mexico.

Brown, Esther L.

2005 Syllable-initial /s/ in Traditional New Mexican Spanish: Linguistic factors favoring reduction 'ahina'. Southwest Journal of Linguistics, 24, 1-2, 13-30.

Brown, Esther L.

2006 The effects of discourse context on phonological representation in memory. Paper presented at the 80th Annual Meeting of the Linguistic Society of America (LSA), Albuquerque, NM. January 5-8.

Brown, Esther L. and Raymond, William D.

In press How discourse context shapes the lexicon: Explaining the distribution of Spanish f- /h- words. Diachronica 20.2

Brown, Esther L. and Torres Cacoullos, Rena

2002 Qué le vamoh aher?: Taking the syllable out of Spanish /s/ reduction. In D. Johnson and T. Sanchez (Eds.), University of Pennsylvania Working Papers in Linguistics: Papers from NWAV 30 (pp. 17-32). Philadelphia: University of Pennsylvania Press.

Bybee, Joan B. 2001

Phonology and Language Use. Cambridge, UK: Cambridge University Press.

Bybee, Joan B. 2002

Word frequency and context of use in the lexical diffusion of phonetically conditioned sound change. Language Variation and Change, 14, 261-290.

Cohn, Abigail C., Brugman, Johanna, Crawford, Clifford and Joseph, Andrew

2005 Lexical frequency effects and phonetic duration of English homophones: An acoustic study. Journal of the Acoustical Society of America, 118, 2036.
De Jong, Kenneth

The supraglottal articulation of prominence in English: Linguistic stress as localized hyperarticulation. Journal of the Acoustic Society of America, 97, 491-504.

Ernestus, Mirjam, Lahey, Mybeth, Verhees, Femke and Baayen, R. Harald

2006 Lexical frequency and voice assimilation. Journal of the Acoustical Society of America, 120, 1040-1051.

File-Muriel, Richard

2009 The role of lexical frequency in the weakening of syllable-final lexical /s/ in the Spanish of Barranquilla, Colombia. Hispania, 92, 348-360.

Forster, Kenneth I. and Chambers, Susan M.

1973 Lexical access and naming time. Journal of Verbal Learning and Verbal Behavior, 12, 627-635.

Fosler-Lussier, Eric and Morgan, Nelson

1999 Effects of speaking rate and word frequency on pronunciations in conversational speech. Speech Communication, 29, 137-158.

Fowler, Carol and Housum, Jonathan

1987 Talkers' signaling of 'new' and 'old' words in speech and listeners' perception and use of the distinction. Journal of Memory and Language, 26, 489-504.

Gahl, Susanne

2008

Time and thyme are not homophones: The effect of lemma frequency on word duration in spontaneous speech. Language, 84, 474-496.

Gahl, Susanne and Garnsey, Susan M.

$2004 \quad$ Knowledge of grammar, knowledge of usage: syntactic probabilities affect pronunciation variation. Language, 80, 748-775.

Gerlach, Birgit and Grijzenhout, Janet

$2000 \quad$ Clitics from different perspectives. In Gerlach and Grijzenhout (Eds), Clitics in Phonology, Morphology and Syntax. Amsterdam: John Benjamins.

Gries, Stefan Th.

2009 Quantitative corpus Linguistics with $r$ : A practical introduction. New York: Routledge.

Guy, Gregory R.

1991 Explanation in variable phonology: An exponential model of morphological constraints. Language Variation and Change, 3, $1-22$.

Jurafsky, Daniel, Bell, Alan, Gregory, Michelle and Raymond, William D.

Probabilistic relations between words: Evidence from reduction in lexical production. In J. Bybee and P. Hopper (Eds.), Frequency and the emergence of linguistic structure. Amsterdam: John Benjamins, pp. 229-254. 
Krug, Manfred

1998

Lipski, John

1984

String frequency: A cognitive motivating factor in coalescence, language processing, and language change. Journal of English Linguistics, 26, 286-320.

für Dialektologie und Linguistik, 51, 31-43.

James and $\mathrm{Li}$, Yingshing

2007 Lexical frequency effects in Taiwan Southern Min syllable contraction. Journal of Phonetics, 37, 21-230.

Pluymaekers, Mark, Ernestus, Mirjam and Baayen, R. Harald

$2005 \quad$ Lexical frequency and acoustic reduction in spoken Dutch. Journal of the Acoustic Society of America, 118, 2561-2569.

Raymond, William D., Dautricourt, Robin and Hume, Elizabeth

2006 Word-medial / $\mathrm{t}, \mathrm{d} /$ deletion in spontaneous speech: Modeling the effects of extra-linguistic, lexical, and phonological factors. Language Variation and Change, 18, 55-97.

Rhodes, Richard A.

1992 Flapping in American English. In W. U. Dressler, M. Prinzhorn and J. Rennison Eds.), Proceedings of the 7th International Phonology Meeting, pp. 217-232. Rosenberg and Sellier.

Schuchart, Hugo

1885

Über die Lautgesetze: gegen die Junggrammatiker. Berlin: R. Oppenheim.

Terrell, Tracy D.

1979

Final /s/ in Cuban Spanish. Hispania, 62, 599-612

Timberlake, Alan

1978 Uniform and alternating environments in phonological change. Folia Slavica, 2, 312-28.

Vigil, Neddy A. 1989

Database for a linguistic atlas of the Spanish of New Mexico and southern Colorado. Computer Methods in Dialectology. Journal of English Linguistics Special Issue, 22, 69-75.

Zipf, George K.

1929

Relative frequency as a determinant of phonetic change. Harvard Studies in Classical Philology, 15, 1-95.

\section{What statistics do learners track? \\ Rules, constraints and schemas in (artificial) grammar learning*}

\section{Vsevolod Kapatsinski}

Rule-based grammatical theories hypothesize that learners of morphophonology pay most attention to typical characteristics of mappings between cells in a morphological paradigm, which can be expressed in rules, rather than to typical characteristics of forms belonging to an individual cell. Bybee (2001) makes the opposite suggestion. The present paper reports data from miniature artificial language learning in the lab suggesting that reliance on product-oriented vs. source-oriented generalizations may depend on the presentation conditions. However, Bybee's position is supported even for presentation conditions that were designed to be maximally favorable for extracting rules.

\section{Introduction}

\subsection{Theoretical background}

All theories of grammar specify the types of generalizations that a human language user relies on in using language productively and thus restrict the human language learner to pay attention only to certain types of patterns in the data to which s/he is exposed. For instance, Chomsky and Halle (1968) and Albright and Hayes (2003), among others, assume reliance on rules. By contrast, Bybee (2001: 128) writes: "[R]ules express sourceoriented generalizations. That is, they act on a specific input to change it in well-defined ways into an output of a certain form. Many, if not all, schemas are product-oriented rather than source-oriented. A product-

* Part of this research was supported by funds from NIDCD Research Grant DC-00111 and NIDCD T32Traning Grant DC-00012 to David Pisoni and the Speech Research Laboratory at Indiana University. Many thanks to Luis Hernandez for technical assistance and to Kenneth deJong, David Pisoni, Robert Port, Linda Smith and the anonymous reviewers for helpful comments. 